\title{
The relationship between incubation and ECS gradient effects'
}

\author{
J. P. J. PINEL 2 AND R. M. COOPER \\ UNIVERSITY OF CALGARY
}

Three groups of rats received electroconvulsive shock (ECS) $10 \mathrm{sec}$., $2 \mathrm{~min}$, or $5 \mathrm{hr}$. after one-trial avoidance training. These $S$ s as well as additional no-ECS control Ss were tested for retention $25 \mathrm{hr}$. afterward. Three more groups received one-trial avoidance training followed by a test of retention $10 \mathrm{sec}, 2 \mathrm{~min}$, or $5 \mathrm{hr}$. later. Both the ECS gradient effect and the incubation effect were demonstrated. The close relationship between the two functions suggested a new interpretation of the ECS gradient effect.

A number of investigators have demonstrated an electroconvulsive shock (ECS) gradient effect, that is, that the sooner an ECS is administered after training the more the performance on a later test of retention is disrupted. Brady (1952) demonstrated a gradient effect after emotional conditioning which occurred over a period of months. On the other hand, Pinel \& Cooper (1966) found a gradient effect after one-trial avoidance training which occurred over a period of hours.

This ECS gradient effect has generally been attributed to an underlying consolidation process; however, the findings of Brady (1951) and Pinel \& Cooper (1966) that their respective passive avoidance tasks incubated or increased in strength over time intervals similar to those over which they were able to demonstrate gradient effects have suggested another interpretation. Possibly, ECS has a greater disruptive effect when administered shortly after learning because the learned response has not had a chance to incubate, and, therefore, is relatively weak. This present experiment was designed to test this hypothesis by attempting to demonstrate both the ECS gradient effect and incubation effect after one-trial avoidance training.

\section{Procedure}

Seven groups of 10 experimentally naive, male, black-hooded rats were trained to drink from a spout located just above a large bar which was invariably depressed when Ss drank. Water was not contingent on a bar press but the bar enabled a record of drinking behavior to be kept. On the last preshock training trial, the median latency, that is, the amount of time it took Ss to depress the bar after being placed in the test box, was $2 \mathrm{sec}$. On the avoidance training day, all Ss received a 12 ma DC shock (calibration resistance $=$ $100 \mathrm{~K})$ the first time they depressed the bar, and then were immediately removed from the box. Three groups of Ss received an ECS $10 \mathrm{sec} ., 2 \mathrm{~min}$., or $5 \mathrm{hr}$. afterward. Convulsions were induced by a 0.3 sec., 50 ma AC current passed via saline-soaked, gauze- covered alligator clips attached to Ss' ears. All Ss were adapted to the ear clips by attaching them briefly after preshock training trials. The three ECS groups plus an additional no-ECS control group were all tested for retention $25 \mathrm{hr}$. afterward. The other three groups received tests of retention at the same intervals at which the ECS groups were receiving an ECS, that is, $10 \mathrm{sec.,} 2 \mathrm{~min}$., or $5 \mathrm{hr}$, after avoidance training. All Ss were deprived of water for 23.5 hr. prior to the last preshock training trial and the postshock test of retention.

\section{Resulis and Discussion}

Figure 1 shows the typical ECS gradient effect. The latencies of the no-ECS control group were significantly longer than both the 10-sec. $(U=13, p<.05)$ and 2-min. $(U=27, p<.05)$ ECS groups. In addition Ss which received an ECS $5 \mathrm{hr}$. after avoidance training had significantly longer latencies than those Ss whose learning-ECS interval was $10 \mathrm{sec}$. $(U=14, p<.05)$.

Figure 2 shows that the avoidance response increased in strength or incubated as the learning-retention interval increased. Rats with a learning retention interval of $5 \mathrm{hr}$. had significantly longer latencies than their 10-sec. $(U=16, p<.05)$ and $2-\min .(U=24, p<.05)$ counterparts.

In Fig. 3 the ECS-gradient and incubation curves are superimposed to show the relationship between the two functions. This close relationship between the gradient and incubation curves supports our hypothesis

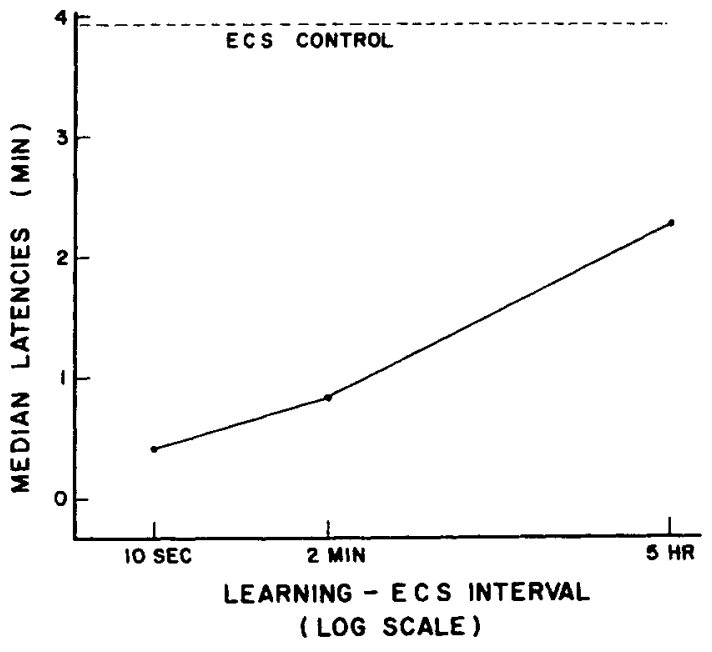

Fig. 1. ECS gradient effect. 


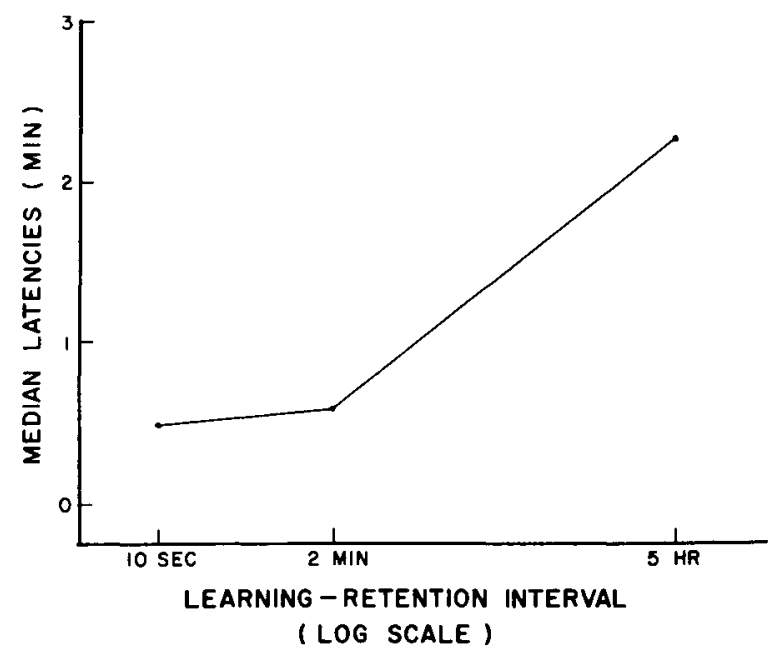

Fig. 2. Incubation efíect.

that the ECS gradient effect may be due to the incubation of the learned response. The present results suggest that as a learned response becomes stronger it is less likely to be disrupted by an ECS, thus resulting in the ECS gradient effect.

On the basis of this study, an important problem in explaining the ECS gradient effect seems to be to identify the process which results in the incubation effect and which in turn underlies the ECS gradient effect.

One interpretation of these results could be that the incubation of the one-trial avoidance response is an outward manifestation of a consolidation process. Although there is no widely accepted detailed statement of the consolidation hypothesis, the essence of this notion is that the storage of a memory becomes more and more stable as time progresses after learning. This notion implies a change in the strength of the storage but no corresponding change in the strength of the response and, therefore, cannot adequately account for the incubation of the one-trial avoidance response.

The incubation effect has been demonstrated by a number of investigators using a variety of avoidancelearning situations (Brady, 1952; Bindra \& Cameron, 1953; and others). This increase in response strength over time has consistently been found in situations that are fear-producing, thus indicating that the incubation of the one-trial avoidance response may be attributable to an increase in fear rather than any change in memory storage.

Although our demonstration that the gradient and

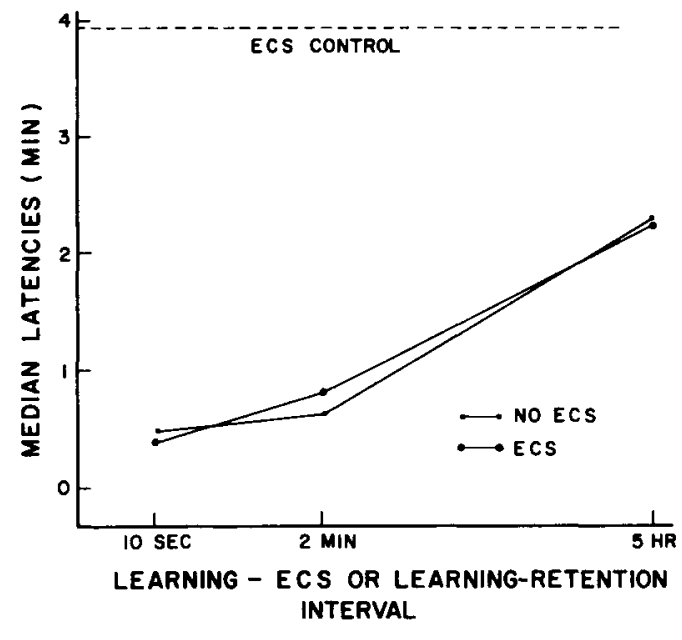

Fig. 3. Relationship between gradient and incubation effects.

incubation curves are almost exactly the same shape is very suggestive, before our hypothesis that the ECS gradient effect is attributable to the incubation or increase in strength of the learned response can be accepted without reservation, the two effects should be shown to be related in other learning situations. For example, Chorover \& Schiller (1965) used a one-trial avoidance task of a different type than used in the present experiment and found an ECS gradient effect to occur over a period of seconds. If our hypothesis is correct, it should be possible to demonstrate that the incubation of this particular one-trial avoidance response occurs over a similar period of time.

\section{References}

Bindra, D., \& Cameron, L. Changes in experimentally produced anxiety with the passage of time: incubation effect. $J$. exp. Psychol., 1953, 45, 197-203.

Brady, J. V. The effect of electroconvulsive shock on a conditioned emotional response: the permanence of the effect. J. comp. physiol. Psychol., 1951, 44, 507-511.

Brady, J. V. The effect of electro-convulsive shock on a conditioned emotional response: the significance of the interval between the emotional conditioning and the electro-convulsive shock. J. comp. physiol. Psychol., 1952, 45, 9-13.

Chorover, S. L., \& Schiller, P. Short-term retrograde amnesia in rats. J. comp. physiol. Psychol., 1965, 59, 73-78.

Pinel, J. P. J., \& Cooper, R. M. Incubation and its implications for the interpretation of the ECS gradient effect. Psychon. Sci., 1966, 6.

\section{Notes}

1. Supported by Canadian National Research Council grant no. APA- 135.

2. Holder of a Canadian National Research Council Studentship. 